\title{
Entwicklungs- und Freigabeprozess automatisierter Fahrzeuge: Berücksichtigung technischer, rechtlicher und ökonomischer Risiken
}

\author{
Thomas Winkle
}

\section{Inhaltsverzeichnis}

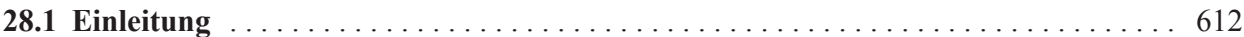

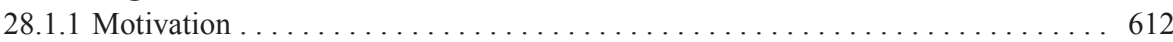

28.1.2 Fragen an die Produktsicherheit zunehmender Fahrzeugautomatisierung . . . . . 612

28.1.3 Technische Weiterentwicklung von Assistenzsystemen -

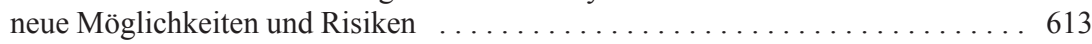

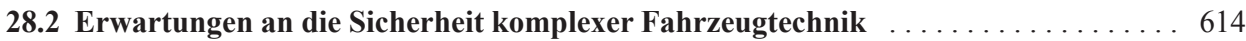

28.2.1 Gesteigerte Verbrauchererwartungen an die Fahrzeugsicherheit . . . . . . . . . . 614

28.2.2 Risiken und Nutzen automatisierter Fahrzeuge . . . . . . . . . . . . . . . . . 615

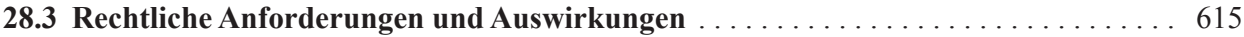

28.3.1 Allgemein anerkannte Regeln der Technik (aaRdT) . . . . . . . . . . . . . . . 617

28.3.2 Produktsicherheitsgesetz (ProdSG) . . . . . . . . . . . . . . . . . . 617

28.3.3 Produkthaftungsgesetz (ProdHaftG) . . . . . . . . . . . . . . . . . . . 618

28.4 Erhöhung der Produktsicherheit automatisierter Fahrzeuge auf der Basis von Expertenerfahrungen aus Haftungs- und Gewährleistungsansprüchen . . . . . . 619

28.4.1 Erfahrungen aus Produktkrisen . . . . . . . . . . . . . . . . . . . . . . . . . . 619

28.4.2 Wesentliche Fragen aus bisherigen Produkthaftungsfällen . . . . . . . . . . . 621

28.4.3 Potenzielle Gefahrensituationen zu Beginn der Entwicklung . . . . . . . . . . . . . 622

28.4.4 Methoden zur Risikobewertung während der Entwicklung . . . . . . . . . 623

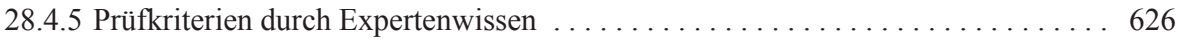

28.4.6 Schritte zur Erhöhung der Produktsicherheit automatisierter Fahrzeuge im allgemeinen Entwicklungsprozess . . . . . . . . . . . . . . . . . . . . 627

28.4.7 Produktbeobachtung nach der Markteinführung . . . . . . . . . . . . . . . 629

28.4.8 Bewertung des Risikos möglicher Funktionsfehler nach der Markteinführung . . 629

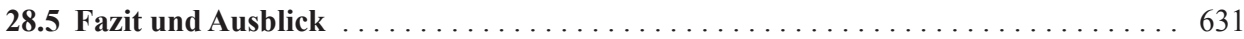

Literatur ........................................ 633

T. Winkle $(\bowtie)$

Technische Universität München - TUM, Maschinenwesen, Lehrstuhl für Ergonomie, Deutschland winkle@carforensic.com 


\subsection{Einleitung}

Die Leistungsfähigkeit von Sensortechnologie und Datenverarbeitung verbessert sich fortlaufend. Dadurch lassen sich eine kontinuierliche Weiterentwicklung von Fahrerassistenzsystemen und die zunehmende Automatisierung von Fahraufgaben bis hin zu selbstfahrenden Fahrzeugen realisieren [1].

\subsubsection{Motivation}

Im Zuge dieser Entwicklung werden technische und elektronische Systeme um ein Vielfaches komplexer und stellen zukünftig Fahrzeughersteller und Entwickler vor neue Herausforderungen. Insbesondere ein Wechsel von der bislang durch Menschen gesteuerten hin zu einer hoch- bzw. vollautomatisierten Fahrzeugführung wirft grundsätzliche Fragen zur Verantwortung oder Haftung auf. Dies erfordert neue Ansätze - in erster Linie neue Sicherheits- und Testkonzepte [2]. Aus rechtlicher Sicht erfordern automatisierte Fahrzeuge zur Minimierung der Risiken sorgfältige Sicherungsmaßnahmen bei der Entwicklung [3]. Diese müssen Nutzer akzeptieren. Laut einem Urteil des Bundesgerichtshofs (BGH) müssen sie auch nach dem jeweils neuesten Stand der Wissenschaft und Technik - innerhalb der Grenzen des technisch Möglichen und wirtschaftlich Zumutbaren - konstruktiv möglich sein sowie geeignet und genügend erscheinen, um Schäden zu verhindern [4].

\subsubsection{Fragen an die Produktsicherheit zunehmender Fahrzeugautomatisierung}

Medienberichte über automatisierte Forschungsfahrzeuge von Automobilherstellern, Zulieferern und IT-Unternehmen weisen seit Jahren auf die Vorbereitung einer Serienentwicklung hin. Für eine Markteinführung fehlen jedoch noch mehrere Voraussetzungen. Eine zunehmende Automatisierung von Fahrfunktionen erfordert modernste, hochkomplexe Technologien. Insbesondere durch die Nutzung elektrischer/elektronischer Komponenten muss mit unvorhersehbaren Reaktionen gerechnet werden, die im Extremfall auch zu Schäden an Leib und Leben führen können. Aktuell zieht eine Vollautomatisierung der Fahraufgaben bei fahrerlosen Fahrzeugen (vgl. [3]) ohne einen verantwortlichen Fahrer als Rückfallebene aufgrund der zunehmenden Komplexität schwer überschaubare Risiken nach sich. Dazu kommen neue Haftungsfragen und eine begrenzte Toleranz bei technischen Ausfällen. Während in Deutschland zurzeit jährlich über 3000 Unfalltote im Straßenverkehr gesellschaftlich offenbar akzeptiert sind, fehlt womöglich jegliche Toleranz bei einem Unfalltoten, der im Zusammenhang mit vermeintlichen technischen Fehlern steht. Obwohl eine Automatisierung des Fahrens ein erhebliches Sicherheitspotenzial verspricht, wird eine übergreifende Vermarktung fahrerloser Fahrzeuge erst erfolgen, wenn eine Klärung der Haftungsfragen und der Verantwortung bei technisch verursachten Schäden 
erfolgt ist. Gesellschaftliche Akzeptanz entsteht u. a. erst dann, wenn der wahrgenommene individuelle Nutzen die erlebten Risiken deutlich überwiegt.

Eine vertiefte Analyse der zu beachtenden Risiken automatisierter Fahrzeuge auf der Basis langjähriger Forschungs- und Produkthaftungserfahrungen liefert Grundlagen für die Vorbereitung ihrer zukünftigen Großserienentwicklung und Vermarktung. Daraus leiten sich Empfehlungen zur Sicherheitsbewertung ab. Bislang sind unter anderem folgende Fragen ungelöst:

- Welche Anforderungen sind für eine Entwicklung und Vermarktung sicherer automatisierter Fahrzeuge zu berücksichtigen?

- Wie sicher ist sicher genug?

- Unter welchen Bedingungen ist ein automatisiertes Fahrzeug fehlerhaft?

- Wie wird die Sorgfaltspflicht bei der Entwicklung sichergestellt?

\subsubsection{Technische Weiterentwicklung von Assistenzsystemen - neue Möglichkeiten und Risiken}

Aus technischer Sicht können automatisierte Fahrzeuge bereits heute Fahraufgaben im Verkehrsgeschehen selbstständig übernehmen. Aktuelle Serienfahrzeuge mit optimierter Sensor-, Computer- und Fahrwerkstechnik ermöglichen zunehmend leistungsfähigere Assistenzsysteme. Einige der heute angebotenen Fahrerassistenzsysteme warnen bei erkannten Gefahren im Längs- oder Querverkehr (z.B. Fahrstreifenverlassenswarnung, Kollisions-, Fahrstreifenwechsel, Nachtsicht- oder Kreuzungsassistent). Andere greifen in die Längs- oder Querdynamik ein (z.B. Antiblockiersystem - ABS, Electronic Stability Control - ESC, Adaptive Cruise Control - ACC). Park-Lenk-Assistenten ermöglichen erhöhten Komfort durch Lenk- und Bremseingriffe im Niedergeschwindigkeitsbereich. Diese teilautomatisierten Fahrzeugsysteme mit temporärer Längs- und Querführungsassistenz werden heute in Serienfahrzeugen ausschließlich unter Annahme der Kontrollierbarkeit eines aufmerksamen Fahrers angeboten. Die Überwachung durch den Fahrzeugführer ist erforderlich. Somit sind Systemeinschränkungen oder -ausfälle dieser Advanced Driver Assistance Systems - ADAS während des normalen Betriebs an den und außerhalb der Systemgrenzen über den Nachweis der Beherrschbarkeit durch den Fahrer abgedeckt (vgl. $[5,6])$.

Beim vollautomatisierten Fahren hingegen steht der Fahrer nicht mehr als Rückfallebene für technische Systemeinschränkungen und Fehler zur Verfügung. Dieser Ersatz menschlich eigenverantwortlichen Handelns durch eine programmierte Maschine bringt technische und rechtliche Risiken sowie Herausforderungen an die Produktsicherheit mit sich. Jedoch lassen sich zukünftige Erwartungen an fahrerlose Fahrzeuge - auch in einer möglichen Umbruchsituation - nur anhand bisheriger Erfahrungen beschreiben. Deshalb werden im Folgenden Analogien auf der Grundlage vergangener und aktueller Erwartungen an die Fahrzeugsicherheit gezogen. 


\subsection{Erwartungen an die Sicherheit komplexer Fahrzeugtechnik}

\subsubsection{Gesteigerte Verbrauchererwartungen an die Fahrzeugsicherheit}

Vollautomatisierte Fahrzeuge müssen am heute weltweit hohen Niveau des Verbraucherbewusstseins für Mängel an Kraftfahrzeugen gemessen werden. Seit dem Jahr 1965 entwickelte sich, verstärkt durch das Buch Unsafe at Any Speed: The Designed-In Dangers of the American Automobile, zunehmend ein kritisches Bewusstsein gegenüber der Automobilindustrie [7, 8]. In dieser Veröffentlichung wirft der Autor Ralph Nader den Fahrzeugherstellern Einsparungen bzw. Sorgfaltspflichtverletzungen zulasten einer sicheren Konstruktion und Herstellung vor. Die Darstellung von Sicherheits- bzw. Konstruktionsmängeln bei General Motors und anderen Herstellern schreckte die Öffentlichkeit auf. Im Nachgang gründete Nader das „Center for Study of Responsive Law“, das Kampagnen gegen die Big Three der US-Autobauer, den Volkswagen-Konzern und andere Automobilkonzerne startete. In der Folge wurden technische Konzepte überarbeitet und optimiert. Im Mittelpunkt von Naders Kritik stand der Chevrolet Corvair. Unter anderem kritisierte Nader die unsichere Fahrdynamik durch den Heckmotor sowie die Pendelachse. Diese veränderte beim Aus- bzw. Einfedern den Sturz der Räder (Neigung gegenüber der Senkrechten). Durch eine Konstruktionsänderung mit einer mehrfach gelagerten Achse bleibt dagegen die Neigung weitgehend gleich, was zu einem stabileren Fahrverhalten führt. Später stand aus ähnlichen Gründen der VW Käfer wegen seiner Seitenwindempfindlichkeit in der Kritik. Auch er war mit Heckmotor und Pendelachse ausgerüstet. Als technische Verbesserung löste der VW Golf mit Frontmotor und stabilerem Fahrverhalten (Markteinführung 1974) den VW Käfer ab.

Neben der Entwicklung neuer und fahrsicherer Fahrzeugkonstruktionen wurde als eine weitere Folge dieser Kritik im Jahr 1970 die US National Highway Traffic Safety Administration (NHTSA) gegründet. Angesiedelt innerhalb des Department of Transportation - auf der Grundlage des „Highway Safety Act of 1970“ - verbessert sie die Straßenverkehrssicherheit. Sie hat es sich zur Aufgabe gemacht, Menschenleben zu schützen, Verletzungen vorzubeugen und Fahrzeugunfälle zu reduzieren. Weiterhin bietet sie dem Verbraucher fahrzeugspezifische Sicherheitsinformationen, die vorher nicht allgemein zugänglich waren. Darüber hinaus begleitet die NHTSA bis heute zahlreiche Untersuchungen automobiler Sicherheitssysteme. Sie hat u.a. die verpflichtende Einführung eines automatisiert eingreifenden Fahrdynamikregelsystems (Electronic Stability Control - ESC) aktiv gefördert. Parallel zu den Aktivitäten der NHTSA zeigt auch die Statistik des Kraftfahrtbundesamtes einen zunehmend sensibleren Umgang mit sicherheitsrelevanten Mängeln durch die Begleitung oder Anordnung von Rückrufaktionen [9]. Mittlerweile bestehen höchste Erwartungen an die Sicherheit von Kraftfahrzeugen. Das äußert sich in einer umfangreichen Sicherheitsausstattung, die heute fast weltweit in jedem Serienfahrzeug erwartet wird. Dazu gehören u. a. das Antiblockiersystem (ABS), Airbags oder eine Fahrdynamikregelung bzw. Electronic Stability Control. Die Häufigkeit von Rückrufaktionen hat zugenommen, obwohl die allgemeine Zuverlässigkeit und Funktionssicherheit 
von Personenkraftwagen gleichzeitig deutlich gesteigert werden konnte. Dauertests in Fachzeitschriften wie Auto Motor und Sport zeigen: Eine Distanz von 100.000 Kilometern ist immer häufiger ohne Panne, außerplanmäßigen Werkstattaufenthalt, defektes Funktionsteil und ohne jeglichen geringfügigen Defekt zu bewältigen.

\subsubsection{Risiken und Nutzen automatisierter Fahrzeuge}

Automatisierte Fahrzeuge werden gesellschaftlich wohl nur dann akzeptiert, wenn der wahrgenommene Nutzen (abhängig vom Wirkungsgrad: Driver versus Robot) gegenüber den erwarteten Risiken (abhängig vom Automatisierungsgrad: Wirkfeld versus Effektivfeld) überwiegt. Zur Verringerung der Risiken führen Hersteller eine Unfalldatenanalyse und ein entsprechendes Risikomanagement durch (s. Abb. 28.1).

Für Automobilhersteller und Zulieferer sind automatisierte Fahrzeuge eine Produktinnovation mit Marktpotenzial. Bei Investitionsentscheidungen und Markteinführungen sind jedoch schwer kalkulierbare Risiken zu bewerten:

- Wird das Markenimage nachhaltig geschädigt, wenn das automatisierte Fahrzeug nicht den Verbrauchererwartungen entspricht?

- Welche Mängel können zu Rückrufaktionen führen?

- Welches Risiko von Produkthaftungsansprüchen besteht, wenn das automatisierte Fahrzeug nicht den Anforderungen eines sicheren Produkts entspricht?

\subsection{Rechtliche Anforderungen und Auswirkungen}

Die gesellschaftlichen und individuellen Erwartungen an die technische Perfektion von Fahrzeugen steigen. So verlangen zunehmende Anforderungen an Fahrzeugqualität und Funktion entsprechend hohe Sicherheitsmaßstäbe bei einer Einführung automatisierter Fahrzeuge. Dies zeigt sich beispielsweise durch die Zunahme von Rückrufaktionen trotz

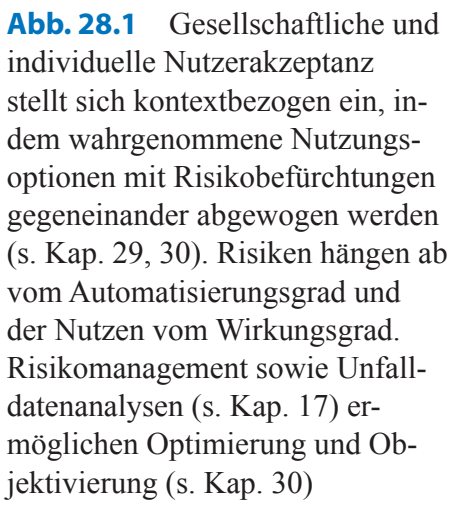

Abb. 28.1 Gesellschaftliche und individuelle Nutzerakzeptanz stellt sich kontextbezogen ein, indem wahrgenommene Nutzungsoptionen mit Risikobefürchtungen gegeneinander abgewogen werden (s. Kap. 29, 30). Risiken hängen ab vom Automatisierungsgrad und der Nutzen vom Wirkungsgrad. Risikomanagement sowie Unfalldatenanalysen (s. Kap. 17) erjektivierung (s. Kap. 30)

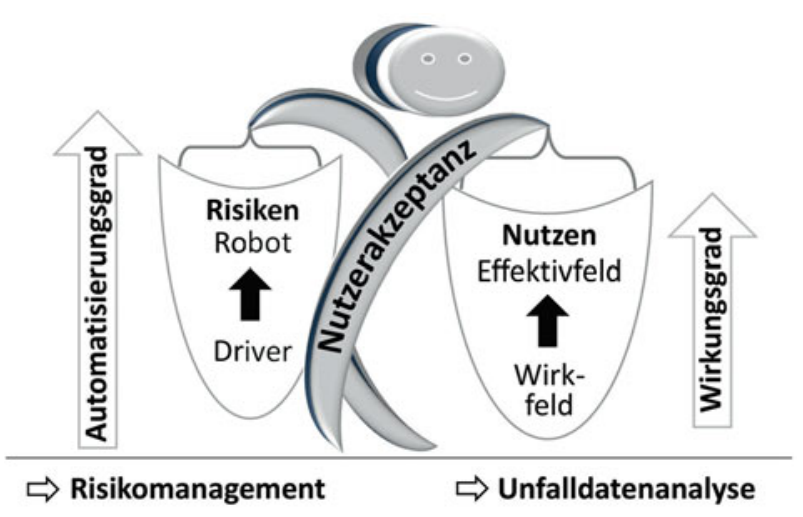


steigender Fahrzeugzuverlässigkeit oder zusätzliche Vorgaben zur Durchführung übergreifender Sicherheitskampagnen (z.B. Motor Vehicle Safety Defects and Recalls Campaigns) oder neue Dokumentationspflichten seitens der Behörden. Ein Beispiel ist das Bundesgesetz „Transportation Recall Enhancement, Accountability and Documentation (TREAD) Act" in den Vereinigten Staaten [10], das eine Reihe neuer und weitergehender Pflichten zur Aufbewahrung von Unterlagen und Meldungen an die Verkehrssicherheitsbehörde National Highway Traffic Safety Administration (NHTSA) einführte. Gleichzeitig werden menschliche Fehler im Straßenverkehr individuell sanktioniert, ohne dass das Verkehrssystem an sich infrage gestellt wird.

Hochkomplexe Technologien und abweichende Definitionen verlangsamen eine Einführung automatisierter Fahrzeuge. Hinzu kommen verschiedenartige technische Vorgaben im interdisziplinären Kontext. In der Vergangenheit konnten sich Entwickler nachvollziehbar an Vorgaben, wie den ,allgemein anerkannten Regeln der Technik“, der „Branchenüblichkeit“ oder dem „Stand der Technik“ orientieren. Der Bundesgerichtshof (BGH) wollte mit seiner Entscheidung vom 16.06.2009 die Anforderungen deutlich erhöhen und prägte für die Automobilindustrie überraschend den Begriff vom neuesten „Stand von Wissenschaft und Technik“. Dies stellt Entwickler vor zusätzliche Herausforderungen. Funktionen, die heute in Forschungsfahrzeugen zu wissenschaftlichen Zwecken unter Laborbedingungen möglich sind, erfüllen bei Weitem nicht die Erwartungen an ein Serienfahrzeug, wie beispielsweise Schutz vor Kälte, Hitze, Erschütterung, Wasser oder Schmutz.

Aus der Sicht eines Entwicklers werden diese rechtlichen Anforderungen an eine sorgfältige Entwicklung neuer komplexer Systeme erst nach definierten Absicherungstests erfüllbar. Diese sollten idealerweise international einheitlich abgestimmt sein. Das BGHUrteil von 2009 begründet die Entwicklungsanforderung - vorbehaltlich der wirtschaftlichen und technischen Eignung zum Serieneinsatz - mit ,... allen konstruktiv möglichen Sicherheitsvorkehrungen ..." unter dem „Stand von Wissenschaft und Technik“ [4] auf der Basis eines Beweissicherungsgutachtens. Dieses fordert jedoch Ultraschallsensoren als Redundanz bei der Erkennung von relevanten Objekten zur Airbagauslösung. Es sei möglich, , ... Ultraschallsensoren rund um das Fahrzeug anzubringen, die den Kontakt mit einem Gegenstand sensieren und die zusätzlich zu den bereits bestehenden Sensoren vor der Auslösung der Airbags abgefragt würden..." [4].

Dieses Beweissicherungsgutachten ist jedoch aus technischer Sicht mehr als fragwürdig, denn aktuelle Sensorausführungen ermöglichen in Serienfahrzeugen nur eine Reichweite von wenigen Metern. Daher ist nach dem heutigen Stand der Technik eine Anwendung der Ultraschallsensorik auf die Erfassung statischer Umgebungen bei langsamer Fahrt im Bereich der Einparkassistenz beschränkt. Die hochfrequenten Schallwellen der Sensoren können durch andere Ultraschallquellen wie Druckluftbremsen von Lkw und Bussen oder Presslufthämmern gestört werden und zu Fehldetektionen führen. Ebenso erfolgt bei schlecht reflektierenden Oberflächen keine Reflektion. Damit bleibt eine Erkennung von Gegenständen gänzlich aus [11]. Außerdem stellte sich in dem Verfahren letztendlich heraus, dass das betroffene Sensorsystem entsprechend der technischen Spezifikation fehlerfrei gearbeitet hat. 
Auch fordert das vorangehende Grundsatzurteil des BGH, Risiken und Nutzen vor einer Markteinführung zu prüfen:

Erforderlich sind die Sicherungsmaßnahmen, die nach dem im Zeitpunkt des Inverkehrbringens des Produkts vorhandenen neuesten Stand der Wissenschaft und Technik konstruktiv möglich sind ... und als geeignet und genügend erscheinen, um Schäden zu verhindern. [4] Sind bestimmte mit der Produktnutzung einhergehende Risiken nach dem maßgeblichen Stand von Wissenschaft und Technik nicht zu vermeiden, ist unter Abwägung der Risiken, der Wahrscheinlichkeit ihrer Verwirklichung und des mit dem Produkt verbundenen Nutzens zu prüfen, ob das gefährliche Produkt überhaupt in den Verkehr gebracht werden darf. [4]

\subsubsection{Allgemein anerkannte Regeln der Technik (aaRdT)}

Eine Auslegung des Rechtsbegriffs ,,allgemein anerkannte Regeln der Technik (aaRdT)“ als Grundlagenregel prägte ein Urteil des Reichsgerichts (RGSt 44, 86) von 1910 auf Basis einer Entscheidung aus dem Jahr 1891 in einem Strafverfahren zu § 330 StGB im Zusammenhang mit dem Baurecht:

Als allgemein anerkannte Regeln der Technik sind solche anzusprechen, die sich aus der Summe aller Erfahrungen im technischen Bereich ergeben, deren Bewährung in der Praxis feststeht und von deren Richtigkeit die Fachleute überzeugt sind.

In verschiedenen Rechtsbereichen haben sie unterschiedliche Bedeutungen. Bei den allgemein anerkannten Regeln der Technik handelt es sich bezogen auf die Produkthaftung um Mindestanforderungen, deren Nichteinhaltung dafür spricht, dass die erforderliche Sicherheit nicht erreicht wurde. Sie sind u. a. in DIN-VDE-Bestimmungen, DIN-Normen, Unfallverhütungsvorschriften und VDI-Richtlinien beschrieben [12].

\subsubsection{Produktsicherheitsgesetz (ProdSG)}

Das Produktsicherheitsgesetz (ProdSG) in der Neufassung vom 08.11.2011 stellt Regeln zu Sicherheitsanforderungen und Verbraucherprodukten auf. Sein Vorläufer war das Geräte- und Produktsicherheitsgesetz vom 01.05.2004, das wiederum das Produktsicherheitsgesetz (ProdSG) vom 22.04.1997 und das Gerätesicherheitsgesetz (GSG) vom 24.06.1968 abgelöst hatte. Der $\S 3$ beschreibt allgemeine Anforderungen an die Bereitstellung von Produkten auf dem Markt:

Ein Produkt darf ... nur auf dem Markt bereitgestellt werden, wenn es bei bestimmungsgemäßer oder vorhersehbarer Verwendung die Sicherheit und Gesundheit von Personen nicht gefährdet. [13] 


\subsubsection{Produkthaftungsgesetz (ProdHaftG)}

Unabhängig von der rechtlichen Anspruchsgrundlage bezeichnet der Begriff „Produkthaftung“ die gesetzliche Haftung des Herstellers für Schäden aus einem fehlerhaften Produkt. Hersteller ist, wer ein Endprodukt, ein Teilprodukt, einen Grundstoff herstellt oder seinen Namen bzw. seine Marke am Produkt anbringt. In Deutschland bestehen für die Produkthaftung zwei unterschiedliche Anspruchsgrundlagen: Die verschuldensabhängige Haftung nach $\S 823$ des Bürgerlichen Gesetzbuchs (BGB) [13] und zum anderen die verschuldensunabhängige Haftung nach dem Produkthaftungsgesetz. So beschreibt das Produkthaftungsgesetz (ProdHaftG - Gesetz über die Haftung für fehlerhafte Produkte) vom 15.12.1989 die Konsequenzen eines Fehlers in $\S 1$ :

Wird durch den Fehler eines Produkts jemand getötet, sein Körper oder seine Gesundheit verletzt oder eine Sache beschädigt, so ist der Hersteller des Produkts verpflichtet, dem Geschädigten den daraus entstehenden Schaden zu ersetzen. [14]

Unabhängig davon, ob der Produktfehler vorsätzlich oder fahrlässig herbeigeführt wird, definiert das ProdHaftG einen Fehler gemäß $§ 3$ Abs. 1 wie folgt:

Ein Produkt hat einen Fehler, wenn es nicht die Sicherheit bietet, die unter Berücksichtigung aller Umstände, insbesondere seiner Darbietung, des Gebrauchs, mit dem billigerweise gerechnet werden kann, sowie des Zeitpunkts, in dem es in den Verkehr gebracht wurde, berechtigterweise erwartet werden kann. [14]

Falls durch ein fehlerhaftes Produkt Schäden entstehen, regelt das Produkthaftungsgesetz die Haftung des Herstellers. Zunächst zieht dies mögliche Ansprüche aus der zivilrechtlichen Haftung für Sachschäden, Vermögensschäden, Personenschäden oder Schmerzensgeld nach sich. Haftpflichtig ist primär der Hersteller. In begründeten Fällen können auch Zulieferer, Importeure, Vertriebshändler oder Verkäufer unbegrenzt haftbar gemacht werden. Darüber hinaus können bei einer begründeten strafrechtlichen Haftung speziell auch Konsequenzen für die Unternehmensleitung oder einzelne Mitarbeiter entstehen, wenn nachweisbar versäumt wurde, Risiken auf ein akzeptiertes Maß zu reduzieren (S. 624, Abb. 28.3). Im Fall von grobem Verschulden bzw. je nach Delikt auch bei Fahrlässigkeit kann dies unter Umständen für einen Entwickler persönlich strafrechtliche Konsequenzen nach sich ziehen.

Neben den rechtlichen Folgen müssen Hersteller auch mit erheblichen negativen wirtschaftlichen Konsequenzen rechnen. Negative Schlagzeilen in den Medien können zu beträchtlichen Umsatz- bzw. Gewinneinbußen, Imageverlust und in der weiteren Folge zum Verlust von Marktanteilen führen. So sind bei der Entwicklung neuer Systeme die Risiken rechtlicher wie auch wirtschaftlicher Folgen zu bedenken. Abbildung 28.2 gibt einen Überblick über mögliche Auswirkungen von Fehlern automatisierter Fahrzeuge. 


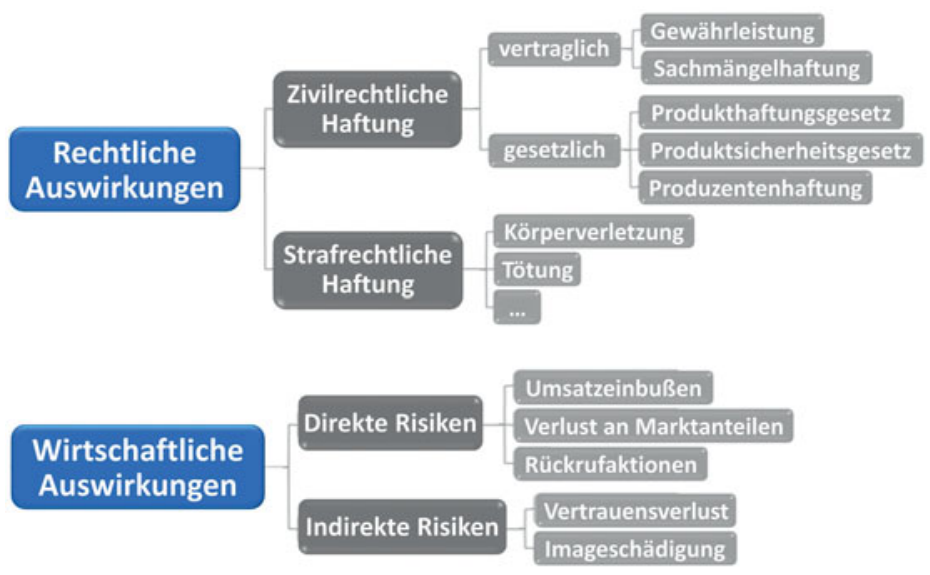

Abb. 28.2 Mögliche Auswirkungen bei Fehlern automatisierter Fahrzeuge

\subsection{Erhöhung der Produktsicherheit automatisierter Fahrzeuge auf der Basis von Expertenerfahrungen aus Haftungs- und Gewährleistungsansprüchen}

\subsubsection{Erfahrungen aus Produktkrisen}

Sichere automatisierte Fahrzeuge werden zukünftig weiterhin von integrierten Qualitätsmanagementsystemen $[15,16]$ und sicheren Interaktionen [17] abhängig sein. Oft waren in der Vergangenheit insbesondere fortschrittliche und erfolgreiche Fahrzeuge von Produktkrisen betroffen.

\subsubsection{Fehlerhafte Zulieferteile}

Folgende Beispiele dokumentieren, wie Teile eines Zulieferers Auslöser für weitreichende Produktkrisen geworden sind.

Der Ford Explorer galt als weltweit meistverkauftes Sport Utility Vehicle. Im Mai 2000 kontaktierte die NHTSA in den USA die beiden Konzerne Ford und Firestone wegen eines auffällig häufigen Auftretens von Reifenschäden durch die Ablösung von Laufflächen. Betroffen waren der Ford Explorer, Mercury Mountaineer und Mazda Navajo. Alle waren werkseitig mit Firestone-Reifen ausgestattet. Nach Reifenschäden kam es bei höheren Geschwindigkeiten zu Schleuderunfällen mit Überschlägen und tödlichem Ausgang. So standen Firestone-Reifen auf Ford-Explorer-Fahrzeugen im Zusammenhang mit über 200 tödlich verletzten Personen in den USA und mehr als 60 Toten in Venezuela. Im gerichtlichen Vergleich bezahlten Ford und Firestone 7,85 Millionen Dollar. Insgesamt betrugen die Schadenersatz- und Strafzahlungen 369 Millionen Dollar. Zum teuren Rückruf von mehreren Millionen Reifen wurden zusätzlich im Anschluss Fehler in der Krisenkommunikation begangen: Die verantwortlichen Manager schoben sich gegenseitig öffentlich die 
Schuld zu. So zerbrach die über 100 Jahre alte freundschaftliche Geschäftsbeziehung der beiden Unternehmen. Bereits 1895 hatte Harvey Firestone Reifen an Henry Ford für die Produktion seines ersten Automobils verkauft. Im weiteren Verlauf der Krise kam es zu eklatanten Imageschäden mit Einbrüchen der Verkaufszahlen für beide Parteien [18].

Einen weiteren Beispielfall für mangelhafte Zulieferteile gab General Motors (GM) im Februar 2014 bekannt. Durch die Folgen der Wirtschaftskrise 2009 stand der Automobilkonzern kurz vor der Insolvenz und hatte nach einer staatlichen Rettungsaktion erstmals wieder Gewinne ausgewiesen sowie Preise für neue Modelle erhalten. Doch der Schalter des Zündschlosses einiger Modelle war scheinbar seit 2001 zu schwach ausgelegt, weshalb der Zündschlüssel während der Fahrt in die Position „Aus“ zurückspringen konnte. In diesem Fall schalten sich Motor, Bremskraftverstärker, Servolenkung und die Airbags ab. Die GM-Ingenieure waren mit dem Vorwurf konfrontiert, den Sicherheitsmangel trotz früher Warnzeichen mehr als zehn Jahre lang ignoriert zu haben. Der Konzern wurde deshalb bereits wegen eines verschleppten Rückrufs zu einer Geldstrafe von 35 Millionen Dollar verurteilt und sieht sich nach Massenrückrufen über Jahre mit milliardenschweren Schadenersatzklagen von Unfallopfern und Fahrzeugbesitzern konfrontiert [19].

\subsubsection{Angeblich selbstbeschleunigende Fahrzeuge}

Fahrzeuge mit automatisiertem Eingriff in die Längs- und Querführung bergen erhebliche Risiken und eine Angriffsfläche für die Behauptung, es sei unerwünschtes Lenk- oder Beschleunigungs- bzw. Verzögerungsverhalten aufgetreten. Mit dem Vorwurf der ungewollten Beschleunigung (unintended acceleration) durch angebliche technische Mängel standen in der Vergangenheit bereits einige Automobilhersteller im Kreuzfeuer der Medien. Vor allem in den USA hätten Fahrzeuge selbstständig beschleunigt und dadurch tödliche Unfälle verursacht. Betroffene Fahrer lösten deshalb Klagewellen aus, die seit Jahrzehnten andauern.

Ein Beispiel hierfür waren die Vorwürfe gegen Toyota, einem weltweit erfolgreichen und für Qualität bekannten Konzern. Toyota schnitt bei einer Kundenzufriedenheitsstudie des amerikanischen Marktforschungsunternehmens J.D. Power and Associates in den Jahren 2002, 2004 und 2005 sehr gut ab. Doch 2009 war Toyota mit Vorwürfen wegen angeblich selbstbeschleunigender Fahrzeuge konfrontiert. Auslöser waren zunächst einzelne Vorfälle von verrutschten Fußmatten, die für ein Verklemmen des Gaspedals verantwortlich gewesen sein sollen. Dann wurde behauptet, die Fahrzeuge hätten wegen mechanisch klemmender Gaspedale während der Fahrt unerwünscht beschleunigt. Da Toyota aus Sicht der NHTSA nicht ausreichend schnell auf die Vorwürfe reagiert hatte, warf man dem Unternehmen eine Verschleierung von Sicherheitsproblemen im Zusammenhang mit mehr als 50 Todesfällen in den USA vor. Neben den Zahlungen zum Schadensausgleich musste Toyota 2010 eine außergewöhnlich hohe Geldbuße von 16,4 Millionen Dollar an die Behörde bezahlen. Daraufhin folgten weitreichende Rückrufaktionen und Schadensersatzklagen [20].

Ein weiteres Beispiel für einen nachgewiesenen technischen Fehler, der zu unerwünschten Beschleunigungen führte, zeigt eine Rückrufaktion der NHTSA vom Juni 2014. Die Adaptive Cruise Control von Geländefahrzeugen der Firma Chrysler reduzierte die Geschwindigkeit nach einem vorübergehenden Übertreten nicht wieder auf die gesetzte Geschwindigkeit, 
sondern beschleunigte unerwünscht weiter. Unfallopfer waren keine zu beklagen. Die kurzfristig eingeleitete Rückrufaktion beschränkte sich auf 6082 Fahrzeuge [21].

\subsubsection{Wesentliche Fragen aus bisherigen Produkthaftungsfällen}

Eigene Erfahrungen des Verfassers aus bisherigen Produkthaftungsfällen haben gezeigt, dass eine strukturierte Entwicklung als Mindestanforderung insbesondere auch für ein sicheres automatisiertes Fahrzeug vorausgesetzt wird (S. 627, Abschnitt 28.4.6). Im Schadensfall sind die im Folgenden genannten Fragen zur Abwehr zivil- und strafrechtlicher Ansprüche von Bedeutung:

- Wurde bereits vor Beginn einer neuen Produktentwicklung unter Abwägung der Risiken, der Eintrittswahrscheinlichkeit und des Nutzens geprüft, ob das Fahrzeug mit dieser technischen Umsetzung überhaupt in den Verkehr gebracht werden darf?

Grundsätzlich existieren neben den allgemeinen gesetzlichen Anforderungen bis heute keine abgestimmten und harmonisierten Methoden für vollautomatisierte Fahrzeuge. Generieren lassen sich diese durch international anerkannte Entwicklungsleitfäden mit Checklisten ähnlich dem mit der ISO 26262 [22] (Teil 3, Concept phase, Seite 24, Controllability) verlinkten RESPONSE 3-ADAS Code of Practice für die Entwicklung und Validierung von Advanced Driver Assistance Systems (ADAS) mit aktiver Längs- und Querführung [5]:

- Welche Maßnahmen wurden zur Risiko-, Schadens- und Gefahrenminimierung über den gesetzlichen Rahmen hinaus ergriffen?

Zukünftige Vorgaben werden sich an den heute gültigen Anforderungen orientieren und diese zum größten Teil übernehmen. Die Methoden zur Bewertung des Risikos während der Entwicklung (S. 623, Abschnitt 28.4.4) gewährleisten, dass bei der Nutzung des Fahrzeugs keine intolerablen Personengefährdungen zu erwarten sind. Deshalb sind bei der Entwicklung mindestens die heute allgemein gültigen Anforderungen, Richtlinien, Verfahren und Prozesse zu berücksichtigen:

- Wurden beispielsweise allgemein anerkannte Regeln, Normen oder technische Vorschriften eingehalten?

Die Einhaltung gängiger Vorgaben allein ist im Allgemeinen nicht ausreichend. Vielmehr werfen sie folgende Fragen auf:

- Wurde mit der geforderten Sorgfalt entwickelt, produziert und vertrieben?

- Hätte der entstandene Schaden mit einer anderen Konstruktion vermieden oder in seiner Auswirkung gemindert werden können?

- Wie verhält sich bzw. wie hätte sich ein Fahrzeug aus dem Wettbewerb verhalten?

- Hätten Warnungen den Schaden verhindern können? 
Ob ein automatisiertes Fahrzeug die erforderliche Sicherheit erreicht hat, zeigt sich am Ende des Entwicklungsprozesses:

- Wurde eine angemessene Sicherheit durch geeignete und genügende Maßnahmen nach dem aktuellen Stand von Wissenschaft und Technik bei Inverkehrbringen erreicht?

Auch nach einer erfolgreichen Markteinführung ist eine Beobachtung im Serieneinsatz zwingend erforderlich. Selbst dann, wenn alle rechtlichen Anforderungen, Richtlinien und Qualitätsprozesse für einen sicheren Gebrauch der entwickelten automatisierten Funktionen und für mögliche Fehlfunktionen eingehalten wurden. Die Beobachtungspflicht ergibt sich aus der Verkehrssicherungspflicht im Rahmen von $\S 823$ Abs. 1 Bürgerliches Gesetzbuch (BGB) [13], deren Verletzung eine Haftung für einen Fehler auslöst, der solchermaßen hätte erkannt werden müssen. So stellt sich bei Produkthaftungsfällen auch die abschließende Frage:

- Wird bzw. wurde das automatisierte Fahrzeug im Kundenbetrieb beobachtet?

\subsubsection{Potenzielle Gefahrensituationen zu Beginn der Entwicklung}

Alltagserfahrungen unserer technologisch fortgeschrittenen Gesellschaft zeigen: Risiken und gefahrenträchtige Handlungen gehören zwangsläufig zum Leben. Unsicherheit und Unwägbarkeiten werden nicht mehr als schicksalhaft hinzunehmende Ereignisse betrachtet, sondern als mehr oder weniger kalkulierbare Unsicherheiten [23]. Daraus ergeben sich höhere Ansprüche an das Risikomanagement für die Hersteller neuer Technologien.

Eine strukturierte Analyse der Gefahren unter Berücksichtigung aller möglichen Umstände kann helfen, einen ersten Gesamtüberblick über die potenziellen Gefahren zu erhalten. Deshalb ist es im frühen Entwicklungsstadium sinnvoll, eine vollständige Beschreibung des automatisierten Fahrzeugs zu geben, um eine logische Gefahrenanalyse und eine anschließende Risikoklassifizierung zu gewährleisten (s. Abschnitt 28.4.4).

Auf dieser Basis ist es möglich, zum Projektstart in einem interdisziplinären Expertenteam (S. 627, Abb. 28.4) gemeinsam eine vollständige Liste der potenziell gefährlichen Situationen zu erstellen. Dies führt in der Regel zu vielen relevanten Situationen. Aufgrund praktischer Erwägungen sollten die Szenarien später für eine Expertenbewertung und Testung auf jene Situationen beschränkt werden, welche die höchste Relevanz besitzen (z. B. relevante Szenarien aus der Verknüpfung verfügbarer Verkehrsunfall-, Wetterdaten und Verkehrssimulationen, s. Kap. 17).

Nach der Systemdefinition ist es empfehlenswert, zunächst Situationen in einer Liste oder Tabelle zu sammeln. Diese berücksichtigt:

- wann die Funktion der Automatisierung verlässlich gewährleistet sein sollte (normale Funktion) 
- in welchen Situationen die Automatisierung verwendet werden könnte, wofür sie jedoch nicht ausgelegt ist (Falschinterpretation und potenzieller Fehlgebrauch)

- wann Leistungsgrenzen für erforderliche Redundanzen erreicht sind

- ob gefährliche Situationen durch Fehlfunktionen der Automatisierung verursacht werden (Versagen)

Die gemeinsame Erarbeitung einer maximalen Anzahl an systemrelevanten Situationen macht es wahrscheinlich, dass keine relevante Gefahr ausgelassen oder vergessen wurde. Im nächsten Schritt empfiehlt sich eine Zusammenfassung von Gefahren mit direktem Einfluss auf die Sicherheit. Nach einer Reduktion auf tatsächliche sicherheitsrelevante Situationen werden hierfür technische Lösungen entwickelt.

\subsubsection{Methoden zur Risikobewertung während der Entwicklung}

In einer Veröffentlichung der Bundesregierung wird im Zusammenhang mit dem Ausstieg aus der Atomenergie erwähnt, dass die deutsche Gesellschaft als „Schicksalsgemeinschaft“ und als Teil der „Weltrisikogesellschaft“ den Fortschritt und Wohlstand bei gleichzeitig beherrschbaren Risiken wünscht [24]. Dies ist sicherlich nur eingeschränkt auf den Straßenverkehr übertragbar, denn die Risiken durch automatisierte Fahrzeuge beschränken sich - im Gegensatz zur Atomenergie - auf einen überschaubaren Personenkreis. Ähnlich sind jedoch spezifische Anforderungen an die verwendeten Methoden zur Analyse und Bewertung der Risiken. Im Folgenden werden fünf gängige Methoden vorgestellt.

\subsubsection{Gefahrenanalyse und Risikobewertung}

Der ADAS Code of Practice zur Entwicklung aktiver Längs- und Querführungsfunktionen $[5,6]$ sowie die ISO 26262 Teil 3 zur Funktionssicherheit [22] beschreiben das Vorgehen zur Gefahrenanalyse und Risikobewertung (G\&R). Auch Teile der in den folgenden Abschnitten genannten Methoden (HAZOP, FMEA, FTA, HIL) verweisen auf die G\&R. Ziel der G\&R ist es, potenzielle Gefahren einer Betrachtungseinheit zu identifizieren, einzustufen und Ziele zu definieren. So lassen sich Gefahren vermeiden und die Risiken auf ein gesellschaftlich und individuell akzeptiertes Maß minimieren. Dazu wird eine „Betrachtungseinheit“ bezüglich ihres Einflusses auf die Sicherheit beurteilt und einem Automotive Safety Integrity Level (ASIL) zugeordnet. Eine „Betrachtungseinheit“ (Item) nach ISO 26262 ist definiert als komplexes elektrisches/elektronisches System oder als Funktion, die mechanische Komponenten verschiedener Technologien enthalten kann. Der ASIL wird bestimmt durch eine systematische Auswertung von möglicherweise gefährlichen Situationen und Betriebsbedingungen. Dabei erfolgt eine Bewertung der Unfallschwere [25] im Zusammenhang mit der Auftrittswahrscheinlichkeit. Eine Reduktion auf eine angenommene Hardwareausfall-Fehlerrate (z.B. ASIL D: $<10^{-8} \mathrm{~h}^{-1}$ ) für gesellschaftlich und individuell akzeptierte Risiken (S. 624, Abb. 28.3) erfolgt durch externe Maßnahmen [22]. 


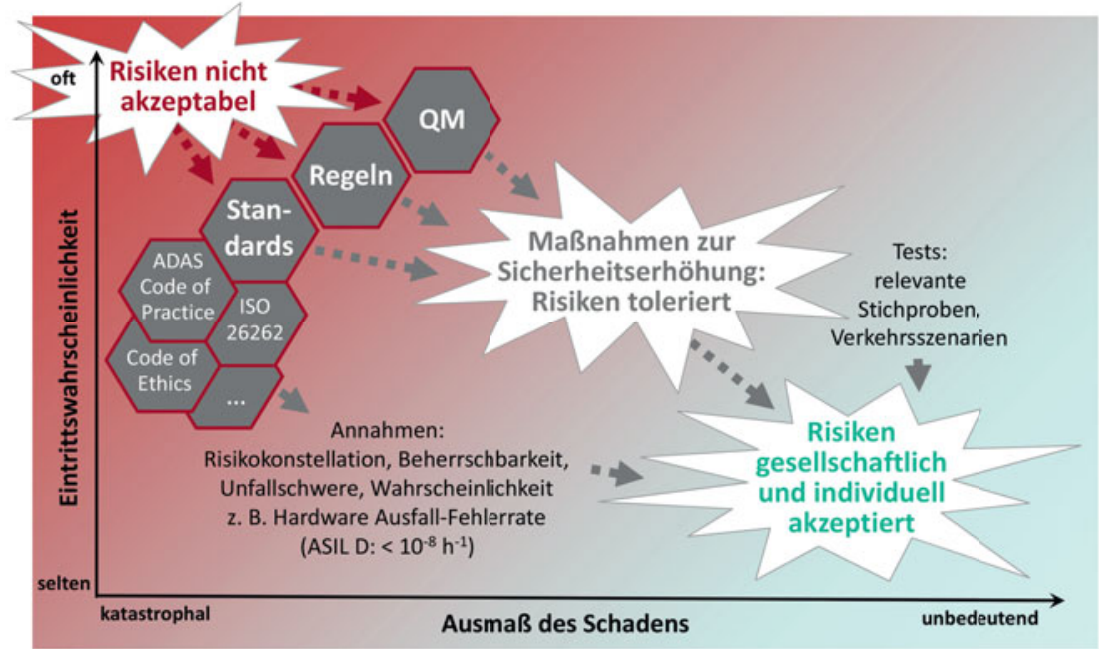

Abb. 28.3 Maßnahmen zur Erhöhung der Sicherheit auf gesellschaftlich und individuell akzeptierte Risiken

Grundlegend kann das Risiko $R$ für einen analytischen Ansatz ausgedrückt werden als Funktion $F$ der Frequenz $f$, mit der ein gefahrbringendes Ereignis auftritt, und der potenziellen Schadensschwere $S$ des sich daraus ergebenden Schadens:

$$
R=F(f, S)
$$

Die Frequenz $f$, mit der ein gefahrbringendes Ereignis auftritt, wird wiederum von verschiedenen Parametern beeinflusst. Ein wesentlicher zu berücksichtigender Faktor ist, wie häufig oder wie lange sich eine Person in einer Situation aufhält, in der eine Gefahr auftreten kann $(E=$ exposure). Ein anderer Faktor, der das Auftreten eines gefahrbringenden Ereignisses beeinflusst, ist die Vermeidung einer potenziellen Schadensauswirkung durch rechtzeitige Reaktionen der am Unfallgeschehen beteiligten Personen bzw. Verkehrsteilnehmer ( $C=$ controllability). Eine Beherrschbarkeit durch den Fahrer entfällt jedoch in Bezug auf das unfallbeteiligte fahrerlose bzw. vollautomatisierte Fahrzeug. Das Produkt E $x C$ ist ein Maß für die Wahrscheinlichkeit, dass in einer gewissen Situation ein Fehler das Potenzial hat, sich entsprechend dem beschriebenen Schaden auszuwirken.

Ein weiterer Faktor ( $\lambda=$ Fehlerrate) lässt sich zurückführen auf unentdeckte zufällige Hardwareausfälle der Systemkomponenten und auf gefährliche systematische Fehler, die im System verblieben sind. Er stellt die Häufigkeit des Auftretens bezogen auf $E$ dar, mit der das automatisierte Fahrzeug selbst ein gefahrbringendes Ereignis auslösen kann. Somit beschreibt das Produkt $f$ die Anzahl der Ereignisse, die während der Zeit $E$ (z. B. gefahrene Kilometer oder Anzahl der Fahrzeugstarts) zu erwarten sind.

$$
f=E \times \lambda
$$


Weiterhin berücksichtigt die ISO 26262 Ausfallraten technischer und elektronischer Bauteile (Failure in Time - FIT). Die Einheit FIT gibt die Anzahl der Bauteile an, die innerhalb von $10^{9}$ Stunden ausfallen. Im Ergebnis aus Auftrittswahrscheinlichkeit $f$ und - falls möglich - Beherrschbarkeit bzw. Kontrollierbarkeit $C$ erfolgt eine Einstufung der „Sicherheitskritikalität" nach ASIL B, C (empfohlene Ausfallwahrscheinlichkeit kleiner $10^{-7}$ je Stunde; dies entspricht einer Rate von 100 FIT) bis ASIL D (geforderte Ausfallwahrscheinlichkeit kleiner $10^{-8}$ je Stunde; dies entspricht einer Rate von 10 FIT). Die höchsten Anforderungen entsprechen dem ASIL D. Zusätzlich berücksichtigt die ISO 26262 neben dem normalen Betrieb des Fahrzeugs auch Serviceanforderungen bis hin zur Stilllegung des Fahrzeugs. In diesem Zusammenhang müssen Entwickler bei der Auswahl von Bauelementen Alterungsfolgen elektronischer Systeme in Betracht ziehen. Steuergeräte oder Sensoren sind durch eine robuste Auslegung entsprechend zu schützen, falls sie mit alterungsempfindlichen Elektrolytkondensatoren zur Energiereserve bestückt wurden. Ein Ausfall darf keine wichtigen Funktionen außer Kraft setzen [22].

\subsubsection{Gefahren- und Operabilitätsstudie (HAZOP)}

Die Gefahren- und Operabilitätsstudie (Hazard and Operability Study - HAZOP) ist eine frühe Risikobewertung, die in der Prozessindustrie entwickelt wurde. Eine HAZOP sucht nach jeder vorstellbaren Prozessabweichung vom normalen Betrieb und betrachtet dann mögliche Ursachen und Konsequenzen. Typischerweise führt ein Spezialistenteam aus den betroffenen Entwicklungsbereichen die Suche bei der HAZOP systematisch durch, um so die Wahrscheinlichkeit zu minimieren, dass wichtige Faktoren übersehen werden [5].

\subsubsection{Fehler-Möglichkeits- und Einfluss-Analyse (FMEA)}

Die Fehler-Möglichkeits- und Einfluss-Analyse (Failure Mode and Effects Analysis FMEA) sowie die integrierte Fehler-Möglichkeits-, Einfluss- und Kritikalitätsanalyse (Failure Mode, Effects and Criticality Analysis - FMECA) sind Methoden zur Zuverlässigkeitsanalyse, die Fehler mit signifikanten Konsequenzen identifizieren, welche die Systemleistung in der betrachteten Anwendung betreffen. Die FMEA basiert auf einem definierten System, einer Baugruppe oder einem Bauteil, für das die grundlegenden Fehlerkriterien (Primärfehlermodi) verfügbar sind. Sie ist eine Technik zur Absicherung und Einschätzung möglicher Fehlerzustände bei der Auslegungsüberprüfung. Sie kann vom ersten Schritt der Systemauslegung bis zum fertigen Produkt angewendet werden. Die FMEA lässt sich auf die Auslegung aller Systemebenen anwenden [26, 27].

\subsubsection{Fehlerbaumanalyse (FTA)}

Die Fehlerbaumanalyse (Fault Tree Analysis - FTA) beschäftigt sich mit der Identifikation sowie Analyse von Bedingungen und Faktoren, die das Auftreten eines definierten Fehlerzustands begünstigen, der die Systemleistung, Wirtschaftlichkeit, Sicherheit oder andere erforderliche Eigenschaften beträchtlich beeinflusst. Der Fehlerbaum ist besonders geeignet für die Analyse komplexer Systeme, die mehrere funktionell zusammenhängende oder unabhängige Untersysteme mit verschiedenen Leistungszielen umfassen. Dies trifft insbe- 
sondere auf Systemauslegungen zu, die eine Zusammenarbeit zwischen vielen spezialisierten technischen Designgruppen erfordern. Beispiele von Systemen, bei denen die Fehlerbaumanalyse verbreitet angewendet wird, sind Kernkraftwerke, Flugzeuge, Kommunikationssysteme, chemische und andere industrielle Prozesse. Der Fehlerbaum selbst ist eine organisierte grafische Darstellung der Bedingungen oder anderer Faktoren, die das Eintreten eines definierten unerwünschten Vorfalls, den man auch Top Event nennt, verursachen oder dazu beitragen [5].

\subsubsection{Hardware-in-the-Loop (HIL)-Tests}

Eine zunehmende Fahrzeugvernetzung stellt besondere Anforderungen an die Absicherung des gesamten Steuergeräteverbunds, z. B. von Bordnetzsicherheit, Buskommunikation, Fahrzeugzustandsmanagement, Diagnose und Flashverhalten. Hardware-in-the-Loop (HIL)-Tests lassen sich anwenden, sobald ein Hardware-Prototyp des Systems oder auch ein Teil davon (z. B. eine elektronische Steuereinheit eines Fahrzeugs) verfügbar ist. In einer software-simulierten, virtuellen Umgebung wird der Prototyp als Device Under Test (DUT) in einen „Loop“ (Kreislauf) eingefügt. Diese soll der echten Umgebung so ähnlich wie möglich sein. Der DUT wird unter Echtzeitbedingungen betrieben [28].

\subsubsection{Prüfkriterien durch Expertenwissen}

Im Freigabeprozess ist eine Vorgehensweise zur Überprüfung vorzusehen. Für den Endnachweis einer Sicherheitsbestätigung automatisierter Fahrzeuge sind Prüfkriterien im Sinne von „,bestanden“ bzw. „nicht bestanden“ zu empfehlen. Unabhängig davon, welche Methoden für den abschließenden Freigabenachweis ausgewählt wurden, sollten sich die Experten darauf einigen, welche Prüfkriterien ausreichend sind, damit das Fahrzeug bestimmte Situationen eines Systemversagens oder Fehlfunktionen erfolgreich bewältigt. Für entsprechende Kriterien sind allgemein anerkannte Werte zum Erreichen der gewünschten Fahrzeugreaktion heranzuziehen. Eine Evaluation kann durch etablierte Methoden erfolgen.

Auf Basis der Liste potenzieller Gefahrensituationen (S. 622, Abschnitt 28.4.3) entwickelt ein Expertengremium die Prüfkriterien für sicheres Fahrzeugverhalten und eventuell auch relevante Testszenarien. Dafür ist insbesondere ein Team von Systemingenieuren und Unfallforschern erforderlich. Die erste Gruppe bietet Wissen über die genauen Systemfunktionalitäten, Zeitabläufe und Erfahrungen mit Fehlermöglichkeiten. Unfallforscher tragen ihr Erfahrungswissen über risikobehaftete Verkehrssituationen bei (s. Kap. 17). Jede bekannte risikobehaftete Situation, in die das Fahrzeug geraten könnte, ist zu berücksichtigen. Zu den identifizierten Risiken spezifiziert der Entwickler mindestens eine mögliche Abhilfemaßnahme im Hinblick auf die Sicherheitsanforderungen. Bezogen auf den abschließenden Nachweis gilt das Testszenario als ,,bestanden“, wenn das automatische Fahrzeug wie erwartet reagiert oder die Situation anderweitig zufriedenstellend löst. 


\subsubsection{Schritte zur Erhöhung der Produktsicherheit automatisierter Fahrzeuge im allgemeinen Entwicklungsprozess}

Für die Gewährleistung der Produktsicherheit automatisierter Fahrzeuge ist ein sorgfältiges Entwicklungskonzept notwendig, welches sich mindestens am aktuellen Stand von Wissenschaft und Technik orientiert. Hierfür wird nachfolgend ein allgemeiner Entwicklungsprozess vorgeschlagen, wie ihn heute vom Grundsatz her mit kleinen Anpassungen die Automobilhersteller für die Entwicklung von Serienfahrzeugen anwenden. Dieser als V-Modell dargestellte generische Entwicklungsprozess (s. Abb. 28.4) bildet eine logische Abfolge der Phasen einer Produktentwicklung und ausgewählte Meilensteine, jedoch nicht zwingend deren Zeitablauf ab [5, 29].

Der Prozess ist eine vereinfachte Darstellung in Form eines V-Modells, das Iterationsschleifen innerhalb der individuellen Entwicklungsphasen gemeinsam mit allen Beteiligten ermöglicht. Innerhalb dieser V-förmigen Prozessstrukturierung (s. Abb. 28.4) sind Elemente des Sicherheitsprozesses berücksichtigt. Auch empfiehlt sich frühzeitig und regelmäßig interdisziplinäre Expertengruppen zu berücksichtigen. Von der Definitionsphase bis zur Absicherung, Abnahme und dem Produktionsstart sollten Experten aus Forschung, (Vor-) Entwicklung, Funktionssicherheit, Produktanalyse, Rechtswesen, Verkehrssicherheit, Technikethik, Ergonomie, Produktion und Vertrieb einbezogen werden.

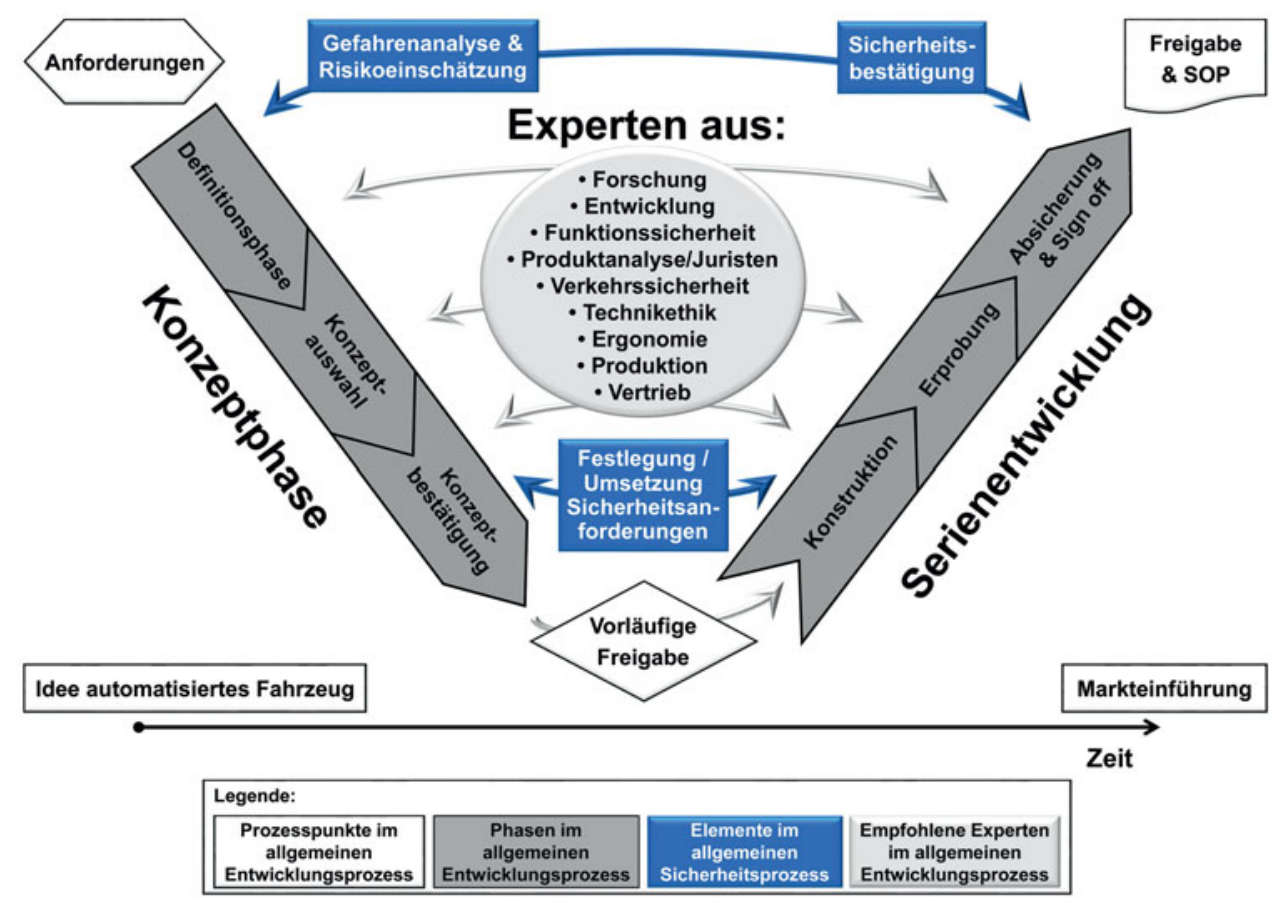

Abb. 28.4 Allgemeiner Entwicklungsprozess für automatisierte Fahrzeuge von der Idee bis zur Markteinführung unter Einbeziehung empfohlener Experten 
Bei den Entwicklungsschritten zur Erhöhung der Produktsicherheit automatischer Fahrzeuge steht die Funktionssicherheit als Schlüsselanforderung im Vordergrund. Sie bezieht sich auf die gesamte Interaktion zwischen dem Fahrzeug und der Umgebung. Eine Interaktion mit dem Fahrer [30] ist dann zu berücksichtigen, wenn hierfür entsprechend dem Anwendungsfall bzw. der Funktionalität eine Schnittstelle vorgesehen ist. Vollautomatische Fahrzeuge schließen im Wesentlichen die fünf folgenden Nutzungssituationen für die Betrachtung der Produktsicherheit ein. Im Vordergrund steht die funktionale Sicherheit des vollautomatischen Fahrzeugs - innerhalb sowie an den Leistungsgrenzen und auch jenseits davon. Weiterhin ist die Funktionssicherheit während und nach Systemausfällen zu betrachten. Eine sorgfältige Entwicklung anwendungssicherer fahrerloser Fahrzeuge muss sicherstellen, dass diese die Kritikalität einer Situation erkennen, über geeignete Maßnahmen zur Gefahrenabwehr (z.B. Degradation, Fahrmanöver) mit einer Rückführung in einen sicheren Zustand entscheiden und diese Maßnahmen schließlich durchführen.

Einen Überblick über einen möglichen Arbeitsfluss im Hinblick auf die Freigabe bis hin zur Stilllegung gibt Abb. 28.5. Am Ende der Entwicklung eines automatisierten Fahrzeugs entscheidet das Entwicklungsteam, ob noch ein abschließender Bestätigungstest erforderlich ist. Dieser bestätigt, dass der Serienstand eine ausreichende Sicherheit erreicht. Hierzu verifiziert das Entwicklungsteam, dass ein Fahrzeug in relevanten Szenarien so wie vorher eingeschätzt oder in einer anderen situativ angemessenen Weise reagiert. Die hierfür verwendeten Daten können aus Methoden zur Bewertung des Risikos während

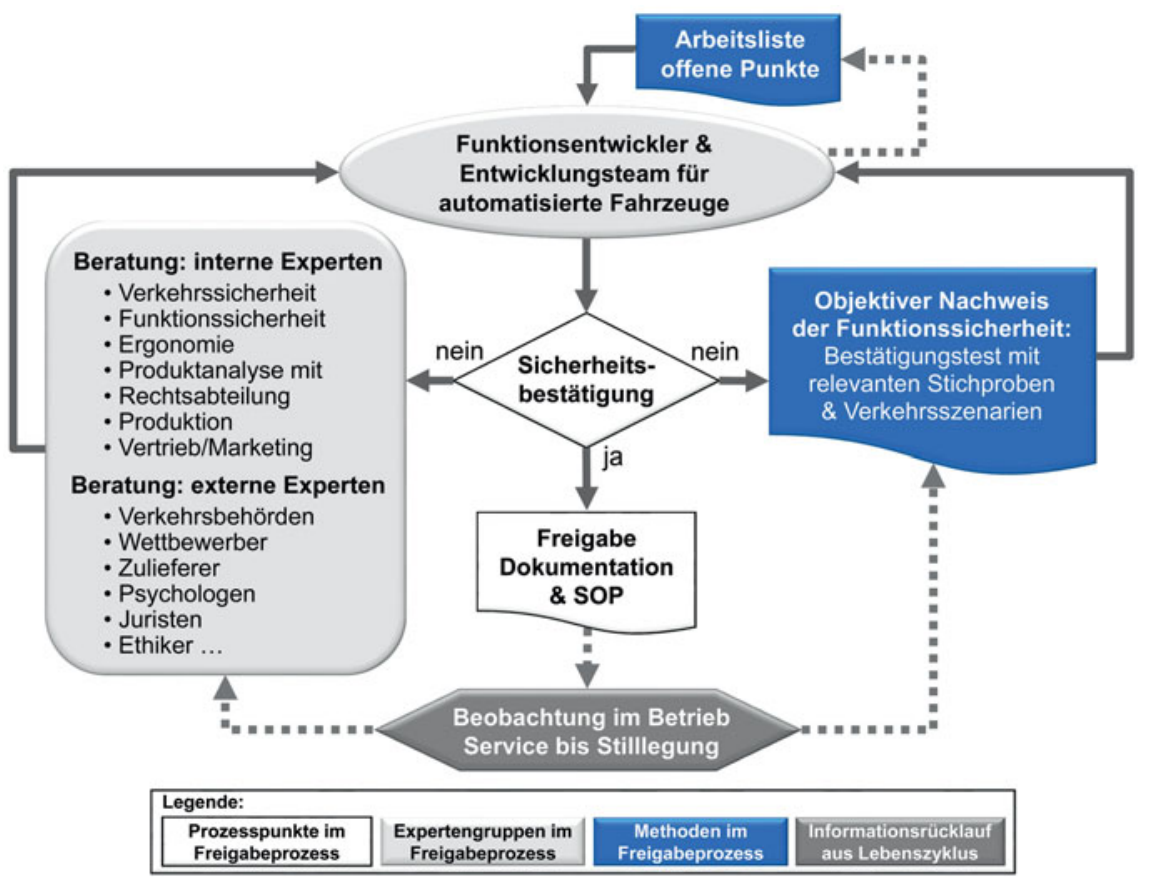

Abb. 28.5 Empfohlener Freigabeprozess für automatisierte Fahrzeuge 
der Entwicklung stammen (z.B. Gefahren- und Risikoanalyse). Drei gleichwertige Freigabewege sind möglich. Eine direkte Freigabe erfolgt durch die erfahrungsbasierte Empfehlung des Entwicklungsteams. Weiterhin kann eine abschließende Sicherheitsbestätigung nach entsprechender Rückversicherung über ein internes bzw. externes interdisziplinäres Expertenforum oder einen objektiven Nachweis verabschiedet werden. Die Bestätigung der Funktionssicherheit ist mittels Bestätigungstests basierend auf relevanten Verkehrsszenarien (s. Kap. 17) und anderen objektiven Stichproben möglich (s. Abb. 28.5).

Das Entwicklungsteam wählt für jedes einzelne Szenario einen geeigneten Weg aus. Ein gemischter Ansatz ist ebenfalls möglich. Wenn das Entwicklungsteam die Sicherheitsbestätigung der Systemauslegung abschließend veranlasst, kann die endgültige Freigabe erteilt werden (vgl. [5]).

\subsubsection{Produktbeobachtung nach der Markteinführung}

Im Anschluss an eine sorgfältige Entwicklung ist ein Hersteller verpflichtet, ein automatisiertes Fahrzeug nach dem sogenannten Inverkehrbringen zu beobachten, um bislang unbekannte Gefahren zu erkennen und nachträglich geeignete Sicherheitsmaßnahmen zu ergreifen. Danach ist ein Automobilhersteller angehalten, mögliche Gefahren zu erkennen (die auch bei nicht bestimmungsgemäßer oder missbräuchlicher Verwendung auftreten können), um mit entsprechenden Maßnahmen wie beispielsweise Rückrufaktionen, Nachbesserung oder Benutzerinformationen zu reagieren.

Als spezielles Beispiel zur Produktbeobachtungspflicht bei Kombinationsrisiken mit Drittzubehör wird unter Produkthaftungsexperten oft ein Urteil des Bundesgerichtshofs (BGH) zitiert. Modellspezifische Motorrad-Lenkerverkleidungen aus dem Zubehör, die amtlich anerkannte Sachverständige einer Prüforganisation im Juni 1977 erstmalig abgenommen hatten, sollen für drei spektakuläre Unfälle verantwortlich gewesen sein, darunter ein tödlicher. Der betroffene Motorradhersteller schrieb am Tag vor dem tödlichen Unfall jeden ihm bekannten Fahrer des betroffenen Modells mit einer Warnung persönlich an. Den Verunglückten erreichte dieses Schreiben indes nicht mehr. Obwohl der Motorradhersteller vor der Verwendung der Verkleidung ausdrücklich warnte, wurde das Unternehmen zu Schadensersatz verurteilt. Der BGH fällte in dieser Sache ein wegweisendes Grundsatzurteil:

Die Firmen müssen künftig nicht nur die Verlässlichkeit ihres Produkts in der Praxis beobachten, sondern vor allem ihre Kunden auf Gefahren im Tagesbetrieb hinweisen - auch auf solche, die durch Verwendung oder Einbau von Zubehör anderer Hersteller entstehen. [31]

\subsubsection{Bewertung des Risikos möglicher Funktionsfehler nach der Markteinführung}

Aufgrund von Vernetzung und Komplexität werden sämtliche Risiken automatisierter Fahrzeuge im Serienbetrieb nur schwer zu überblicken sein. Die zunehmende Sensibilität 


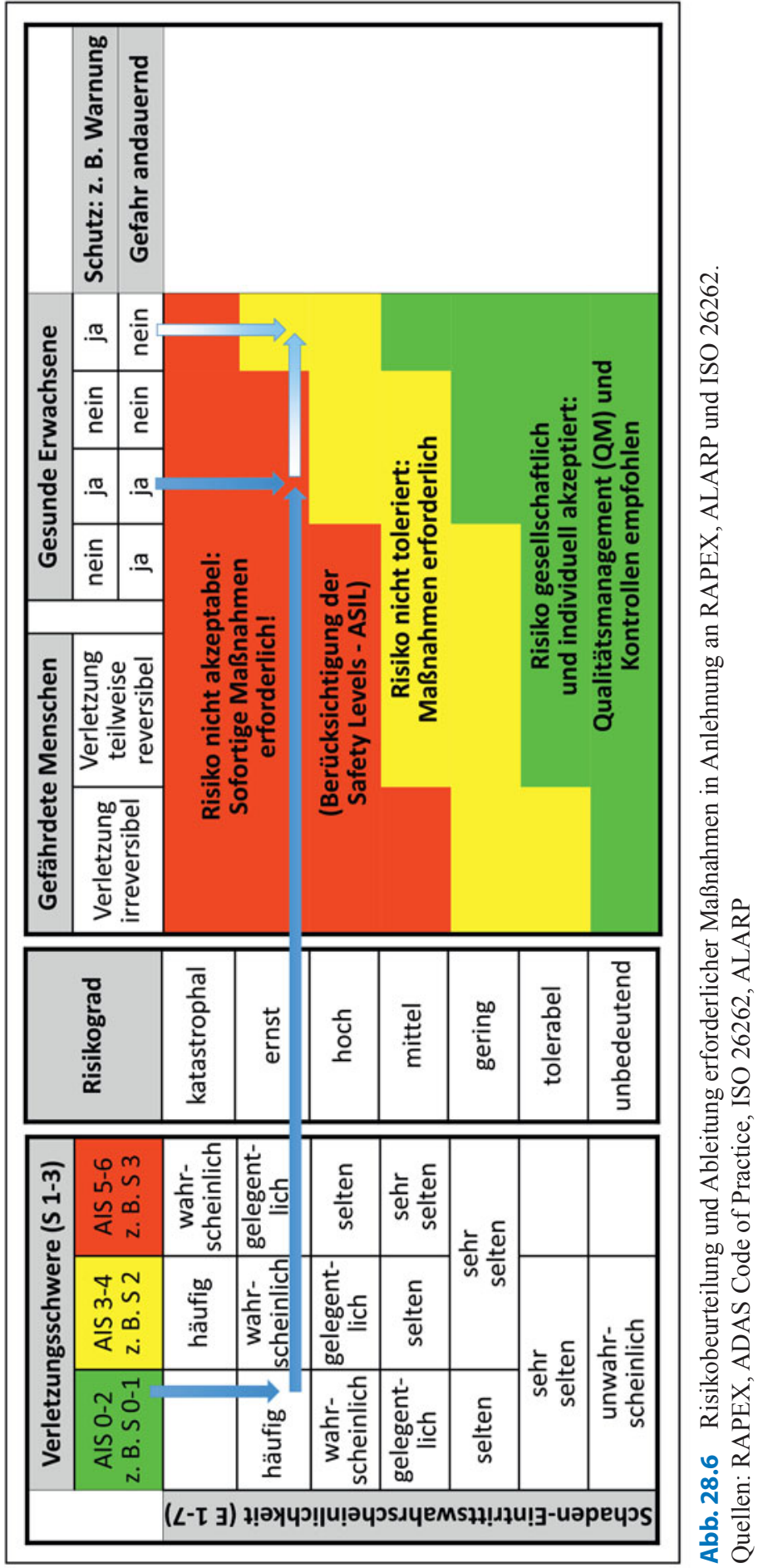


für Mängel zeigt die deutliche Zunahme weltweiter Rückrufaktionen. Tauchen im Serienbetrieb bislang unbekannte Fehler auf, sind für diese gegebenenfalls nach einer Risikobewertung entsprechende Maßnahmen zu ergreifen.

Zur Risikoanalyse und Risikobeurteilung von Produktfehlern nach der Markteinführung - im Hinblick auf die Notwendigkeit bzw. Dringlichkeit von Rückrufaktionen - nutzen die EU und das deutsche Kraftfahrtbundesamt Tabellen des Schnellwarnsystems RAPEX (Rapid Exchange of Information System) [32]. Die Risikoeinstufung erfolgt zunächst über die Verletzungsschwere (Schadensausmaß S beispielsweise nach AIS) und die Eintrittswahrscheinlichkeit eines Schadens - ähnlich dem ALARP-Prinzip (As Low As Reasonable Possible) [33], dem ISO 26262-Standard [22] und dem Code of Practice für ADAS mit aktiver Längs- und Querführung [5, 6]. Daraus wird der Risikograd abgeleitet. Die abschließende Beurteilung der Dringlichkeit von erforderlichen Maßnahmen berücksichtigt ein Verletzungsrisiko der verletzungsgefährdeten Menschen (Einfluss von Alter, körperlicher Gesundheitszustand usw.) bzw. die Gefahr für einen mental gesunden Erwachsenen (Reaktionsfähigkeit, Risikobewusstsein usw.) und die Nutzung von Schutzmaßnahmen wie angemessene Warnhinweise (s. Abb. 28.6).

\subsection{Fazit und Ausblick}

Einerseits sind Erwartungen in der Gesellschaft verständlich, die zunehmend höchste Sicherheitsansprüche an neue Technologien nach „Stand der Wissenschaft und Technik“ fordern. Andererseits verhindert ein unrealistischer Anspruch an die technische Perfektion bzw. das Streben nach 100-prozentiger Fehlerfreiheit gegebenenfalls eine Markteinführung automatisierter Fahrzeuge und damit die Chance auf ein revolutionäres Nutzenpotenzial. Dadurch bleiben Innovationen aus.

Manche bahnbrechende Technologie stünde uns heute nicht zur Verfügung, hätten Vorsicht und Zurückhaltung bei ihrer Einführung die Oberhand gewonnen. Ein Beispiel für eine mutige Innovation zeigte der deutsche Ingenieur und Automobilpionier Carl Friedrich Benz. Bereits im Jahr 1885 absolvierte er mit seinem Prototyp, dem funktionstüchtigen Benz-Patent-Motorwagen, die ersten Testrunden. In seinem Buch Lebensfahrt eines Erfinders erinnert sich Benz an seine ersten Ausflüge:

Hatte ich meine Versuchsfahrten bis dahin mit besonderer Vorliebe weitab von der Stadt vorgenommen - auf dem Fabrikgelände oder draußen auf dem alten, einsamen Wall (Ringstraße), der damals noch um die Stadt Mannheim sich herumzog und fast gar nicht begangen war -, so scheute ich vom Frühjahr 1886 ab die Menschen und ihre Kritik nicht mehr. [34]

Als der Motorwagen allerdings bei einer Panne liegenblieb, erntete Benz Mitleid, Spott und Hohn:

Wie kann man sich in so einen unzuverlässigen, armseligen, lautlärmenden Maschinenkasten setzen. (...) Wenn ich einen solchen Stinkkasten hätte, würde ich zu Hause bleiben. [34] 
Trotz aller Verneinung und Ablehnung als Antwort auf das nächtelange beständige Arbeiten an seiner Lebensaufgabe hielt Benz mit Unterstützung seiner Frau am Glauben an die Zukunft seines Patentwagens fest und wurde damit Wegbereiter für eine der bedeutendsten Innovationen moderner Mobilität. Die Vorbereitung auf Fahrzeuge mit fortgeschrittenem Automatisierungsgrad erfordert ebenfalls eine konsequente Vorgehensweise nach dem Vorbild von Benz.

Auch die Markteinführung hoch- und vollautomatisierter Fahrzeuge muss sich bislang mit Barrieren auseinandersetzen. Die ersten Anbieter am Markt und damit die Pioniere gehen zunächst erhöhte Risiken ein, sodass sich der mögliche gesamtgesellschaftliche Nutzen durch diese neue Technologie nur gemeinsam umsetzen lässt. Homann beschreibt diesen Entscheidungskonflikt bei der Markteinführung durch das entscheidungstheoretische Konzept des sogenannten Gefangenendilemmas. Zur Überwindung der bei hochund vollautomatisierten Fahrzeugen vorliegenden Dilemmasituation müssen die unüberschaubaren Risiken für die Hersteller durch neue institutionelle Arrangements bewertbar und entscheidbar gemacht werden [35]. Vorbehaltlose Information und transparente Politik fördern und forcieren den fachübergreifenden gesellschaftlichen Dialog.

Bis heute muss der Fahrer aufgrund von Zulassungsvorgaben bei Serienfahrzeugen stets seine Hände am Lenkrad behalten und besitzt permanent die Kontrolle über das Fahrzeug. Auch automatisierte Forschungsfahrzeuge sowie absehbare Fahrzeugentwicklungen von IT-Unternehmen, Automobilherstellern und Zulieferern werden in absehbarer Zeit bei komplexen Verkehrssituationen einen Fahrer als verantwortliche Rückfallebene voraussetzen.

Fahrerlose Fahrzeuge hingegen bedeuten den Beginn einer völlig neuen Dimension. Neue Ansätze und Aktivitäten sind unabdingbar [36]. Es gilt, sich an der zukünftigen Möglichkeit des vollautomatisierten Fahrens zu orientieren, aus Mustern der Vergangenheit zu lernen und bisherige Vorgehensweisen innerhalb der technischen Eignung und wirtschaftlichen Zumutbarkeit dem Stand der Wissenschaft bzw. der Technik anzupassen [37].

Neben einer allgemeinen Klärung der Verantwortung für Unfall- und Produktrisiken können möglicherweise neue begleitende Maßnahmen wie Schulungen oder spezielle Fahrzeugbeobachtungen zur erfolgreichen Markteinführung und zum sicheren Betrieb beitragen. Für den erforderlichen Informationsaustausch, die Speicherung von Fahrzeugdaten (beispielsweise Event Data Recorder) und möglichen kriminellen Eingriffen sind abgestimmte technische Schutzvorkehrungen vorzusehen (s. Kap. 25, 30). Neben abgestimmten Datenschutzrichtlinien werden Experten der Technikethik, beispielsweise in einem Code of Ethics, die Einhaltung ethischer Werte sicherstellen (vgl. Abb. 28.3, 28.4, 28.5). Dabei sind Sicherheitsanforderungen im Sinne der grundlegenden ethischen Frage „Wie sicher ist sicher genug?“ zu beantworten. Expertenerfahrungen können Entscheidendes zur Erhöhung der Sicherheit und zur Erfüllung von Kundenerwartungen bei akzeptablen Risiken beisteuern. Vor dem Hintergrund zunehmender Verbraucheranforderungen sind Expertenerfahrungen - insbesondere aus bisherigen Produkthaftungsverfahren - zur Erhöhung der Produktsicherheit im Entwicklungs- und Freigabeprozess unverzichtbar. 
Zur Großserienvermarktung sicherer automatisierter Fahrzeugtechnologien - die sich zudem in einem vielschichtigen Gesamtsystem wiederfinden - sind interdisziplinär abgestimmte Prozesse erforderlich. Eine belastbare Beurteilung der Serienreife verlangt nach neuen harmonisierten Methoden für vergleichbare Sicherheitsnachweise, beispielsweise mittels Simulation relevanter Szenarien aus der Verknüpfung weltweit verfügbarer Unfalldaten (s. Kap. 17). Dies gilt ebenfalls für die Erfüllung gesetzlicher bzw. zulassungsrechtlicher Regelungen, bei der Ermittlung neuer Möglichkeiten zur Risikoverteilung (vgl. [38]) und bei der Schaffung von neuen Entschädigungseinrichtungen. Zum Nachweis der Sorgfaltspflicht im bestehenden Qualitätsmanagementsystem empfiehlt sich die Weiterentwicklung erfahrungsbasierter, international gültiger Richtlinien, Werkzeuge, Methodenbeschreibungen sowie Leitfäden mit Checklisten, die auf dem ADAS Code of Practice aufbauen $[5,39]$ und den praktizierten Stand von Wissenschaft und Technik innerhalb der technischen Eignung und wirtschaftlichen Zumutbarkeit repräsentieren und dokumentieren. Der ADAS Code of Practice wurde zur sicheren Inverkehrbringung künftiger Advanced Driver Assistance Systems mit aktiver Unterstützung der primären Fahraufgabe (Quer-, Längsführung inklusive vollautomatisierter Notbremseingriffe) erarbeitet und 2009 über die European Automobile Manufacturers Association (ACEA) veröffentlicht. Er korrespondiert mit der ISO $26262 \mathrm{zu}$ den Anforderungen der funktionalen Sicherheit von elektrischen, elektronischen und programmierbaren Systemen. Als Entwicklungsleitlinie enthält er auch Empfehlungen für die Analyse und Beurteilung komplexer MenschMaschine-Interaktionen, wie sie im normalen Gebrauchsfall sowie im Fehlerfall auftreten können [5, 6]. Bei unerwarteten Fehlfunktionen, die zukünftig zu größeren Schäden führen können, sind Produktexperten aus der Entwicklung in die Ursachenforschung einzubeziehen und anzuhören. Sachverständige, die nicht direkt in die Entwicklung eingebunden sind, sollten sich die Fachkompetenz für eine fundierte Begutachtung neuer Technologien vor Gericht aneignen.

Automatisiertes Fahren erfordert in der Entwicklung vernetztes, fachübergreifendes Denken mit einem flexiblen und zugleich strukturierten Handlungsraum. Die Entwicklung erschließt bislang eine unbekannte Welt mit vielen Unsicherheiten, die Vorbehalte und Widerstände verursachen können. Für eine erfolgreiche Einführung serienreifer Fahrzeuge bilden in vivo gesammelte Erkenntnisse aus Vergangenheit und Gegenwart die entscheidende Voraussetzung. So wird die Serienreife trotz technischer, rechtlicher und ökonomischer Risiken zum Nutzen der Gesellschaft möglich.

\section{Literatur}

1. Bengler K, Flemisch F (2011) Von H-Mode zur kooperativen Fahrzeugführung - Grundlegende Ergonomische Fragestellungen, 5. Darmstädter Kolloquium: kooperativ oder autonom? Darmstadt

2. Bengler K, Dietmayer K, Färber B, Maurer M, Stiller C, Winner H (2014) Three Decades of Driver Assistance Systems: Review and Future Perspectives, IEEE Intelligent Transportation System Magazine, ISSN 1939-1390, Volume 6, Issue 4, S. 6-22 
3. Gasser T, Arzt C, Ayoubi M, Bartels A, Bürkle L, Eier J, Flemisch F, Häcker D, Hesse T, Huber W, Lotz C, Maurer M, Ruth-Schumacher S, Schwarz J, Vogt W (2012) Rechtsfolgen zunehmender Fahrzeugautomatisierung, Wirtschaftsverlag NW (Berichte der Bundesanstalt für Straßenwesen F83) Bergisch Gladbach

4. Bundesgerichtshof (2009) Zur Haftung eines Fahrzeugherstellers, BGH-Urteil vom 16.06.2009, Az. VI ZR 107/08, Karlsruhe

5. Knapp A, Neumann M, Brockmann M, Walz R, Winkle T (2009) Code of Practice for the Design and Evaluation of ADAS, Preventive and Active Safety Applications, eSafety for road and air transport, European Commission Integrated Project, Response 3, European Automobile Manufacturers Association - ACEA, www.acea.be, Brüssel

6. Donner E, Winkle T, Walz R und Schwarz J (2007) RESPONSE 3 - Code of Practice für die Entwicklung, Validierung und Markteinführung von Fahrerassistenzsystemen (ADAS), In: Technischer Kongress 2007, Verband der Automobilindustrie (VDA), Sindelfingen, S. 231-241

7. Nader R (1965) Unsafe at Any Speed: The Designed-In Dangers of the American Automobile, Grossman Publishers, Inc., New York

8. Nader R (1972) Unsafe at Any Speed: The Designed-In Dangers of the American Automobile, Expanded edition, Grossman Publishers, Inc., New York

9. Kraftfahrtbundesamt Jahresberichte (2014) http://www.kba.de, Flensburg

10. United States of America (2000) Transportation Recall Enhancement, Accountability, and Documentation TREAD Act - H.R. 5164, and Public Law No. 106-414

11. Noll M, Rapps P (2012) Ultraschallsensorik, In: Winner H, Hakuli S, Wolf G (Hrsg.) Handbuch Fahrerassistenzsysteme, 2. Auflage, S. 110-122, Vieweg+Teubner, Wiesbaden

12. Krey V, Kapoor A (2012) Praxisleitfaden Produktsicherheitsrecht, Hanser, 2. Auflage, München

13. Köhler H (2012) BGB Bürgerliches Gesetzbuch, Deutscher Taschenbuch Verlag, 69. Auflage, München

14. European Commission (1985) Council Directive 85/374/EEC of 25 July 1985 on the approximation of the laws, regulations and administrative provisions of the Member States concerning liability for defective products, Brüssel

15. International Organization for Standardization (ISO), ISO 9001 (2015) Quality management systems - Requirements, Genf

16. International Organization for Standardization (ISO), ISO/TS 16949 (2009) Particular requirements for the application of ISO 9001 for automotive production and relevant service part organizations - Functional safety, Genf

17. Akamatsu M, Green P, Bengler K (2013) Automotive Technology and Human Factors Research: Past, Present and Future, In: International Journal of Vehicular Technology, Hindawi Publishing Corporation, Kairo, New York

18. Hartley R F (2011) Management Mistakes and Successes, 25th Anniversary Edition, 1. Auflage, USA 2011, S. 342

19. National Highway Traffic Safety Administration (2014) Recall: Electrical System: Ignition Switch, NHTSA Campaign Number: 14V-047, Report Receipt Date: February 7, 2014, http:// www.nhtsa.gov

20. National Highway Traffic Safety Administration (2014) Additional Information on Toyota Recalls and Investigations, http://www.nhtsa.gov

21. National Highway Traffic Safety Administration (2014) Recall: Forward Collision Avoidance, Adaptive Cruise Control, Vehicle Speed Control, Accelerator Pedal, NHTSA Campaign Number: 14V293000, Report Receipt Date: June 4, 2014, http://www.nhtsa.gov

22. International Organization for Standardization (ISO), ISO 26262 (2011) Road Vehicles - Functional safety, Genf

23. Grunwald A (2013) Handbuch Technikethik, J.B. Metzler, Stuttgart 
24. Merkel A, Töpfer K, Kleiner M, Beck U, Dohnany K, Fischer U, Glück A, Hacker J, Hambrecht J, Hauff V, Hirche W, Hüttl R, Lübbe W, Marx R, Reisch L, Renn O, Schreurs M, Vasssilidis M, Bachmann G, Sauer I, Teuwsen R, Thiel G (2011) Ethik-Kommission Sichere Energieversorgung Deutschlands, Energiewende - Ein Gemeinschaftswerk für die Zukunft, Presse- und Informationsamt der Bundesregierung, S. $24 \mathrm{ff}$, Berlin

25. Association for the Advancement of Automotive Medicine (2005) The Abbreviated Injury Scale (AIS) Update 2008, Barrington IL

26. Werdich M (2012) FMEA - Einführung und Moderation - durch systematische Entwicklung zur übersichtlichen Risikominimierung, 2. Auflage, Springer Vieweg, Wiesbaden

27. Verband Deutscher Automobilhersteller (2006) VDA-Band 4, Qualitätsmanagement in der Automobilindustrie, Sicherung der Qualität vor Serieneinsatz - Produkt- und Prozess-FMEA, 2. Auflage, Frankfurt/Main

28. Heising B, Ersoy M, Gies, S (2013) Hardware-in-the-loop Simulation, In Fahrwerkhandbuch: Grundlagen, Fahrdynamik, Komponenten, Systeme, Mechatronik, Perspektiven, 4. Auflage, S. 574-575, Springer Vieweg, Wiesbaden

29. Maurer M (2012) Entwurf und Test von Fahrerassistenzsystemen. In: Winner H, Hakuli S, Wolf G (Hrsg.) Handbuch Fahrerassistenzsysteme, 2. Auflage, S.43-52, Vieweg Teubner, Wiesbaden

30. Bengler K, Zimmermann M, Bortot D, Kienle M, Damböck D (2012) Interaction Principles for Cooperative Human-Machine Systems. In: Information Technology, Wissenschaftsverlag Oldenburg

31. Bundesgerichtshof (1986) Zur Produktbeobachtungspflicht für Kombinationsrisiken - Gefahrenabwendungspflicht, BGH-Urteil vom 9.12.1986, Az. VI ZR 65/86, Karlsruhe

32. Europäische Union (2010) Amtsblatt L 22 - Entscheidung der Kommission zur Festlegung von Leitlinien für die Verwendung des gemeinschaftlichen Systems zum raschen Informationsaustausch RAPEX gemäß Artikel 12 und des Meldeverfahrens gemäß Artikel 11 der Richtlinie 2001/95/EG über die allgemeine Produktsicherheit, Luxemburg

33. Becker S, Brockmann M, Jung C, Mihm J, Schollinski H-L, Schwarz J, Winkle T (2004) ADAS - from Market Introduction Scenarios towards a Code of Practice for the Development and Evaluation, RESPONSE 2, European Commission, Final Public Report, Brüssel

34. Benz C (1925) Lebensfahrt eines deutschen Erfinders, Die Erfindung des Automobils, Erinnerungen eines Achtzigjährigen, Neuausgabe zur 50-jährigen Erinnerung, Koehler \& Amelang, März 1936, Leipzig

35. Homann K (2005) Wirtschaft und gesellschaftliche Akzeptanz: Fahrerassistenzsysteme auf dem Prüfstand, In: Maurer M, Stiller C (Hrsg.) Fahrerassistenzsysteme mit maschineller Wahrnehmung, S. 239-244, Springer, Berlin, Heidelberg

36. Matthaei R, Reschka A, Rieken J, Dierkes F, Ulbrich S, Winkle T, Maurer M (2015) Autonomes Fahren, In: Winner H, Hakuli S, Lotz F, Singer C (Hrsg.) Handbuch Fahrerassistenzsysteme, 3. Auflage, S. 1146-1168, Vieweg Teubner, Wiesbaden

37. Scharmer O, Kaufer K (2013) Leading from the emerging future - from Ego-System to EcoSystem economies - applying theory $U$ to transforming business, society and self, BerrettKoehler Publishers, San Francisco CA

38. Donner E, Schollinski H-L, Winkle T, Jung C, Dilger E, Kanz C, Schwarz J, Bastiansen E, Andreone L, Becker S, Mihm J, Jarri P, Frost F, Janssen W, Baum H, Schulz W, Geissler T, Brockmann M (2004) Methods for Risk-Benefit-Analysis of ADAS: Micro Perspective and macroscopic socioeconomic evaluation, RESPONSE 2, European Commission Public Report, Project Deliverable D2, Brüssel

39. Becker S, Schollinski H-L, Schwarz J, Winkle T (2003) Introduction of RESPONSE 2, EU Projekt. In: M. Maurer, C. Stiller (Hrsg.), Workshop Fahrerassistenzsysteme - FAS, Leinsweiler 